\title{
FLUID DYNAMICS OF AN EXPANDING IDEAL FLUID*
}

\author{
BY
}

LEIF RONGVED ${ }^{1}$

\begin{abstract}
One studies in this paper the spreading of disturbances superimposed on an infinite ideal fluid region that is everywhere expanding homogeneously and isotropically. All fluid motion studied satisfies Euler's full nonlinear fluid dynamic equations for the ideal adiabatic monatomic gas.

For Fluid motion thus governed one shows that a general transformation exists which transforms any given first fluid motion to a second fluid motion that initially is identical to the superimposition of the first motion and the motion of a homogeneous and isotropic expansion. The purpose of this paper is to describe and compare the expressions for the first and second motions to reveal how the spreading of a disturbance superimposed on an expanding fluid region differs from the spreading of an initially identical disturbance superimposed on a stationary fluid region.
\end{abstract}

0. Introduction. When the homogeneous and isotropic expansion is undisturbed, then each fluid element recedes radially from any other element at a constant rate proportional to the distance between the elements at any given time. Moreover, each fluid element is a possible location of the origin of an inertial reference frame. One shows in Sec. 1 that this expansion is in exact accordance with Euler's equations for all ideal fluids whose pressure is a function of the mass density.

In Sec. 2 one shows that the ideal adiabatic monatomic gas exhibits a unique behavior. Namely, for this fluid all phases of infinitesimal dilatational waves superimposed on the homogeneous and istropic expansion propagate with different constant velocities relative to inertial reference frames. In all other fluids, where the pressure is a function of the mass density, the inertial phase velocities of such superimposed waves are either accelerated or decelerated by the expansion.

For this fluid and this expansion one derives in Sec. 3 a transformation which transforms any given first solultion of Euler's equations to a second one, which initially is identical to the superimposition of the first solution and the expansion. One denotes the first and second solutions for corresponding solutions.

In Sec. 4 one compares aspects of corresponding solutions to describe how various characteristics of a disturbance propagating in the expanding fluid are gradually

\footnotetext{
*Received February 2, 1989.

${ }^{1}$ Retired Member of Technical Staff, AT\&T Bell Laboratories.
} 
changed due to the nonlinear interaction between the motions of the disturbance and the expansion.

All results given in this paper apply also to a homogeneous and isotropic fluid contraction. The transformation given here was obtained fortuitously, and is not known to be related to other subject matters published.

1. The homogeneous and isotropic expansion. Using indicial notation Euler's equations for ideal fluids, when pressure is the only action on fluid elements, are (see, for example, [1, articles 237, 238]

$$
\begin{aligned}
& \rho \frac{D v_{i}}{D t}=-\frac{\partial p}{\partial x_{i}} \\
& \frac{D \rho}{D t}=-\rho \frac{\partial v_{i}}{\partial x_{i}}
\end{aligned}
$$

where $\rho, v_{i}$, and $p$ are in turn the mass density, velocity, and pressure, which are functions of time, $t$, and the cartesian coordinates, $x_{1}, x_{2}$, and $x_{3}$ and

$$
\frac{D}{D t}=\frac{\partial}{\partial t}+v_{j} \frac{\partial}{\partial x_{j}} \text {. }
$$

One can show by substitution in (1.1) to (1.3) that $v_{i}=v_{i}^{*}$ and $\rho=\rho^{*}$, where

$$
\begin{gathered}
v_{i}^{*}=\frac{x_{i}}{t+\bar{t}} \\
\rho^{*}=\frac{\bar{\rho}}{(1+t / \bar{t})^{3}}
\end{gathered}
$$

and where $\bar{t}$ and $\bar{\rho}$ are arbitrary constants, constitute an exact solution for all fluids where $p$ is a function of $\rho$, formally written as

$$
p=g(\rho)
$$

where $g$ is an arbitrary function. In this paper a star superscript indicates any quantity associated with the undisturbed expansion (1.4) and (1.5).

For later use one derives $D v_{i}^{*} / D t$. By (1.3)

$$
\frac{D v_{i}^{*}}{D t}=\frac{\partial v_{i}^{*}}{\partial t}+v_{j} \frac{\partial v_{i}^{*}}{\partial x_{j}}
$$

or by $(1.4)$

$$
\frac{D v_{i}^{*}}{D t}=-\frac{v_{i}^{*}}{t+\bar{t}}+\frac{v_{j} \partial x_{i} / \partial x_{j}}{t+\bar{t}} .
$$

Now, $v_{j} \partial x_{i} / \partial x_{j}=v_{j} \delta_{j i}=v_{i}$, hence

$$
\frac{D v_{i}^{*}}{D t}=\frac{v_{i}-v_{i}^{*}}{t+\bar{t}} .
$$

Since $D v_{i}^{*} / D t=0$, when $v_{i}=v_{i}^{*}$, the velocity of any given fluid element is constant when the expansion is undisturbed. By (1.4) this constant velocity is

$$
v_{i}^{*}=\frac{\bar{x}_{i}}{\bar{t}}
$$

where $\bar{x}_{i}$ is a constant radius vector to the given element at $t=0$. One notes by (1.10) that if $\bar{t}$ is chosen respectively positive and negative, then (1.4) and (1.5) describe an 
expansion and a contraction. By $(1.5)$ one notes that to keep $\rho^{*}$ positive the range of $t$ for the two phases must be respectively $(-\bar{t}, \infty)$ and $(-\infty, \bar{t})$. Moreover, the expansion phase begins at $t=-\bar{t}$ with a singularity in $v_{i}^{*}$ and $\rho^{*}$ at all points, and the contraction phase ends with the same singularities at $t=\bar{t}$. All matters dealt with in this paper apply to both phases. However, to conserve space one refers only to the expansion phase.

It is apparent from (1.10) that each element of the undisturbed expansion is, as mentioned in the introduction, a possible location for the origin of an inertial reference frame. These are called "local reference frames." In all other inertial frames a fixed point in the frame, other than the origin, moves with a fluid element of the undisturbed expansion. Finally, one notes that the mathematical description of the undisturbed expansion is identical in all local reference frames.

2. A special fluid associated with the expansion. If $S_{i}$ is the radius vector of a moving point in a local reference frame, and if $U_{i}$ is its velocity relative to the undisturbed expanding fluid at the point, then its velocity relative to the local reference frame, $d S_{i} / d t$ is given by

$$
\frac{d S_{i}}{d t}=U_{i}+\frac{S_{i}}{t+\bar{t}}
$$

where, by (1.4), the last term in (2.1) is the undisturbed expansion velocity at the point.

There is a unique form of $U_{i}$ that makes $d S_{i} / d t$ a constant. To find it one assumes that $d^{2} S_{i} / d t^{2}=0$, and obtains from $(2.1)$

$$
O=\frac{d U_{i}}{d t}+\frac{1}{t+\bar{t}}\left[\frac{d S_{i}}{d t}-\frac{S_{i}}{t+\bar{t}}\right]
$$

By (2.1) one rewrites (2.2) as

$$
\frac{1}{t+\bar{t}} \frac{d\left[(t+\bar{t}) U_{i}\right]}{d t}=0
$$

By (2.3), the desired form of $U_{i}$ is

$$
U_{i}=\frac{\bar{U}_{i}}{1+t / \bar{t}}
$$

where $\bar{U}_{i}$ is a constant vector. Thus, a point moving with this gradually diminishing velocity relative to the expanding fluid does not accelerate or decelerate relative to inertial space.

The relationship (2.4) reveals a characteristic of wave propagation in the expanding fluid. Namely, if any phase in a train of infinitesimal dilatational waves is to propagate in the expanding fluid without acceleration or deceleration relative to inertial space, then the propagation velocity, $c$ relative to the undisturbed expanding fluid must have the form (2.4), i.e.,

$$
c=\frac{\bar{c}}{1+t / \bar{t}}
$$


where $\bar{c}$ is a constant. There is a unique fluid that has this wave velocity relative to the fluid. (See, for example, [1, article 25, equation 6].) Namely, the fluid for which

$$
\lim _{\rho \rightarrow \rho^{*}} \frac{d p}{d \rho}=c^{2}
$$

The left side of (2.6) is by (1.6) a function of $\rho^{*}$, and then by (1.4), (1.5), and (2.5) the right side of $(2.6)$ can be written as $(\bar{c})^{2} \rho^{* 2 / 3} /(\bar{\rho})^{2 / 3}$. Therefore, one may write

$$
\frac{d p}{d \rho}=\frac{(\bar{c})^{2} \rho^{2 / 3}}{(\bar{\rho})^{2 / 3}}
$$

or

$$
p=\bar{p}\left(\frac{\rho}{\bar{\rho}}\right)^{5 / 3}
$$

where $\bar{p}=\left(\frac{3}{5}\right) \bar{\rho}(\bar{c})^{2}$. This is the constitutive law of the unique fluid, and it is the adiabatic law of an ideal monatomic gas. (See, for example, [2, article 6.6 and table 6.4].) For this fluid one describes next the transformation discussed in the introduction.

3. The Transformation relating corresponding solutions. One proves in this section that if the fluid density and velocity $\widehat{\rho}\left(x_{1}, x_{2}, x_{3}, t\right)$ and $\widehat{v}_{i}\left(x_{1}, x_{2}, x_{3}, t\right)$ are solutions of Euler's equations for the fluid (2.8), then there is a corresponding solution that also satisfies the same equations given by

$$
\begin{gathered}
\rho\left(x_{1}, x_{2}, x_{3}, t\right)=f^{3} \hat{\rho}\left(X_{1}, X_{2}, X_{3}, T\right) \\
v_{i}\left(x_{1}, x_{2}, x_{3}, t\right)=f \widehat{v}_{i}\left(X_{1}, X_{2}, X_{3}, T\right)+v_{i}^{*}\left(x_{1}, x_{2}, x_{3}, t\right)
\end{gathered}
$$

where

$$
\begin{gathered}
X_{i}=f x_{i} \\
T=f t \\
f=(1+t / \bar{t})^{-1} .
\end{gathered}
$$

To simplify the symbolism in a rather lengthy proof to follow, one distinguishes between $\hat{\rho}\left(x_{1}, x_{2}, x_{3}, t\right), \widehat{v}_{i}\left(x_{1}, x_{2}, x_{3}, t\right)$, and $\hat{\rho}\left(X_{1}, X_{2}, X_{3}, T\right), \widehat{v}_{i}\left(X_{1}, X_{2}, X_{3}, T\right)$ without the use of the arguments in the parentheses as follows. One introduces two functions, $R$ and $V_{i}$ defined by

$$
\begin{aligned}
R & =\widehat{\rho}\left(X_{1}, X_{2}, X_{3}, T\right) \\
V_{i} & =\widehat{v}_{i}\left(X_{1}, X_{2}, X_{3}, T\right)
\end{aligned}
$$

and, in this section, when one writes $\hat{\rho}, \hat{v}_{i}$ one means $\hat{\rho}\left(x_{1}, x_{2}, x_{3}, t\right), \widehat{v}_{i}\left(x_{1}, x_{2}, x_{3}, t\right)$, and when one writes $R, V_{i}$ one means $\hat{\rho}\left(X_{1}, X_{2}, X_{3}, T\right), \widehat{v}_{i}\left(X_{1}, X_{2}, X_{3}, T\right)$. Moreover, one dispenses with the arguments, $\left(x_{1}, x_{2}, x_{3}, t\right)$, used for $\rho, v_{i}$, and $v_{i}^{*}$ since these are considered to be functions of the lower case variables throughout. 
One also rewrites Euler's equations for the fluid (2.8) with $\widehat{\rho}, \widehat{v}_{i}$ and obtains from (1.7) and (1.3) and (2.8)

$$
\begin{gathered}
\frac{\widehat{D} \widehat{v}_{i}}{\widehat{D} t}=-\bar{a} \frac{\partial \widehat{\rho}^{2 / 3}}{\partial x_{i}} \\
\frac{\widehat{D} \ln \widehat{\rho}}{\widehat{D} t}=-\frac{\partial \widehat{v}_{i}}{\partial x_{i}} \\
\frac{\widehat{D}}{\widehat{D} t}=\frac{\partial}{\partial t}+\widehat{v}_{j} \frac{\partial}{\partial x_{j}}
\end{gathered}
$$

where $\bar{a}=(\bar{c})^{2} /(\bar{\rho})^{2 / 3}$. For later use one notes that $R$ and $V_{i}$ satisfy the equations

$$
\begin{gathered}
\frac{D V_{i}}{D T}=-\bar{a} \frac{\partial R^{2 / 3}}{\partial X_{i}} \\
\frac{D \ln R}{D T}=-\frac{\partial V_{i}}{\partial X_{i}} \\
\frac{D}{D T}=\frac{\partial}{\partial T}+V_{j} \frac{\partial}{\partial X_{j}} .
\end{gathered}
$$

Equations (3.11) to (3.13) follow from (3.8) to (3.10) simply by exchanging $x_{i}, t$ for $X_{i}, T$ in both the derivatives and in $\hat{\rho}$ and $\widehat{v}_{i}$, an exchange that does not alter the validity of the equations.

One proves now that if $\hat{\rho}$ and $\widehat{v}_{i}$ satisfy (3.8) to (3.10), which implies that $R$ and $V_{i}$ satisfy (3.11) to (3.13), then the corresponding solution, $\rho, v_{i}$, given by (3.1) to (3.5) satisfies

$$
\begin{aligned}
& \frac{D v_{i}}{D t}=-\bar{a} \frac{\partial \rho^{2 / 3}}{\partial x_{i}} \\
& \frac{D \ln \rho}{D t}=-\frac{\partial v_{i}}{\partial x_{i}}
\end{aligned}
$$

where $D / D t$ is given by (1.3), and also the initial conditions

$$
\begin{gathered}
\rho=\widehat{\rho} \quad \text { when } t=0 \\
v_{i}=\widehat{v}_{i}+v_{i}^{*} \quad \text { when } t=0 .
\end{gathered}
$$

One notes first by (3.3) to (3.5), that when $t=0$ then $f=1, X_{i}=x_{i}$, and $T=t$. Therefore, by (3.1) and (3.2) the initial conditions (3.16) and (3.17) are satisfied. To complete the proof one relates next the left and right sides of (3.14) and (3.15) in turn to the corresponding left and right sides of (3.11) and (3.12).

First, for the right side of (3.14) one finds by (3.2) and (3.7)

$$
\frac{D v_{i}}{D t}=\frac{D\left(f V_{i}\right)}{D t}+\frac{D v_{i}^{*}}{D t}
$$

By differentiation, (1.3), and (1.9) one rewrites (3.18) as

$$
\frac{D v_{i}}{D t}=-\frac{f^{2}}{\bar{t}} V_{i}+f \frac{D V_{i}}{D t}+\left(v_{i}-v_{i}^{*}\right) \frac{f}{\bar{t}} .
$$


By (3.2), and (3.7) the first and last term on the right side of (3.19) cancel, and by (1.3) one writes (3.19) as

$$
\frac{D v_{i}}{D t}=f\left(\frac{\partial V_{i}}{\partial t}+v_{j} \frac{\partial V_{i}}{\partial x_{j}}\right)
$$

By (3.3) to (3.5) one writes (3.20) as

$$
\frac{D v_{i}}{D t}=f\left[\frac{\partial V_{i}}{\partial T} \frac{\partial T}{\partial t}+\frac{\partial V_{i}}{\partial X_{j}} \frac{\partial X_{j}}{\partial t}+\left(f V_{j}+v_{j}^{*}\right) \frac{\partial V_{i}}{\partial X_{k}} \frac{\partial X_{k}}{\partial x_{j}}\right] .
$$

Now, $\partial T / \partial t=f^{2}, \partial X_{j} / \partial t=-f v_{j}^{*}$, and $\partial X_{k} / \partial x_{j}=f \delta_{j k}$, hence (3.21) becomes

$$
\frac{D v_{i}}{D t}=f^{3} \frac{\partial V_{i}}{\partial T}-f^{2} v_{j}^{*} \frac{\partial V_{i}}{\partial X_{j}}+f^{3} V_{j} \frac{\partial V_{i}}{\partial X_{j}}+f^{2} v_{j}^{*} \frac{\partial V_{i}}{\partial X_{j}} .
$$

The second and fourth term on the right side of (3.22) cancel, and by (3.13) then

$$
\frac{D v_{i}}{D t}=f^{3} \frac{D V_{i}}{D T} \text {. }
$$

Next, looking at the right side of (3.14), one finds by (3.1) and (3.6)

or by (3.3) and (3.5)

$$
\frac{\partial \rho^{2 / 3}}{\partial x_{i}}=f^{2} \frac{\partial R^{2 / 3}}{\partial x_{i}}
$$

$$
\frac{\partial \rho^{2 / 3}}{\partial x_{i}}=f^{3} \frac{\partial R^{2 / 3}}{\partial X_{i}} .
$$

By (3.25), (3.23), and (3.11) one finds that (3.14) is satisfied.

For the left side of (3.15) one obtains by (3.1) and (3.6)

$$
\frac{D \ln \rho}{D t}=\frac{D \ln R}{D t}-\frac{3 f}{\bar{t}} \text {. }
$$

By (1.3) (3.26) becomes

$$
\frac{D \ln \rho}{D t}=\frac{\partial \ln R}{\partial t}+v_{j} \frac{\partial \ln R}{\partial x_{j}}-\frac{3 f}{\bar{t}} .
$$

By (3.2), (3.7), and (3.3) to (3.5) one obtains

$$
\frac{D \ln \rho}{D t}=f^{2} \frac{\partial \ln R}{\partial T}-f v_{J}^{*} \frac{\partial \ln R}{\partial X_{j}}+\left[f V_{j}+v_{j}^{*}\right] f \frac{\partial \ln R}{\partial X_{j}}-\frac{3 f}{\bar{t}} .
$$

By cancellation and (3.13) one writes (3.28) as

$$
\frac{D \ln \rho}{D t}=f^{2} \frac{D \ln R}{D T}-\frac{3 f}{\bar{t}}
$$

Finally, for the right side of (3.15) one obtains by (3.2) and (3.7)

or by (3.3) to (3.5) and (1.4)

$$
\frac{\partial v_{i}}{\partial x_{i}}=f \frac{\partial V_{i}}{\partial x_{i}}+\frac{\partial v_{i}^{*}}{\partial x_{i}}
$$

$$
\frac{\partial v_{i}}{\partial x_{i}}=f^{2} \frac{\partial V_{i}}{\partial X_{i}}+\frac{3 f}{\bar{t}}
$$

By (3.31), (3.29), and (3.12) one finds that (3.15) is satisfied. This completes the proof of the transformation (3.1) to (3.5) which generates from any solution, $\widehat{\rho}, \widehat{v}_{i}$ its corresponding solution $\rho, v_{i}$. 
4. Expansion effects. The transformation (3.1) to (3.5) is used in this section to describe how the spreading of a disturbance in the expanding fluid differs from the spreading of an initially identical disturbance in the stationary fluid. The two disturbances are denoted corresponding disturbances as mentioned before.

One notes that a disturbance associated with any exact solution of Euler's equations is appropriate, because of nonlinear terms, only when the disturbance is imposed on a stationary fluid with constant mass density, $\bar{\rho}$. Accordingly, $\hat{\rho}-\bar{\rho}$ and $\widehat{v}_{i}$ are appropriate density and velocity fluctuations relative to the stationary fluid. One rewrites (3.1) and (3.2) so that these fluctuations appear on the right sides of the equations. With the aid of (1.5) one obtains

$$
\begin{gathered}
\rho\left(x_{1}, x_{2}, x_{3}, t\right)-\rho^{*}(t)=f^{3}\left[\hat{\rho}\left(X_{1}, X_{2}, X_{3}, T\right)-\bar{\rho}\right] \\
v_{i}\left(x_{1}, x_{2}, x_{3}, t\right)-v_{i}^{*}\left(x_{1}, x_{2}, x_{3}, t\right)=f \widehat{v}_{i}\left(X_{1}, X_{2}, X_{3}, T\right) .
\end{gathered}
$$

One also rewrites (3.3) to (3.5) as

$$
\begin{gathered}
x_{i}=\frac{X_{i}}{f} \\
t=\frac{T}{f} \\
f=1-\frac{T}{\bar{t}} .
\end{gathered}
$$

Note by (3.4) and (3.5) that $(1+t / \bar{t})(1-T / \bar{t})=1$. Thus, (4.5) and (3.5) yield the same value for $f$. Also, the left sides of (4.1) and (4.2) are density and velocity fluctuations relative to the density and velocity, $\rho^{*}$ and $v_{i}^{*}$ of the undisturbed expansion. Moreover, it is apparent that when $t$ or $T$ is zero, then $\rho-\rho^{*}, v_{i}-v_{i}^{*}$ and $\hat{\rho}-\bar{\rho}, \widehat{v}_{i}$ are identical. Thus, (4.1) to (4.5) describe how the spreading of a disturbance in the expanding fluid differs from the spreading of an initially identical disturbance in the stationary fluid.

For disturbances in general one finds by (4.1) to (4.5), when $0<T<\bar{t}$, that fluctuations in $\hat{\rho}-\bar{\rho}$ and $\widehat{v}_{i}$ at the radius vector, $X_{i}$ and time, $T$ are replicated in $\rho-\rho^{*}$ and $v_{i}-v_{i}^{*}$ at the larger radius vector, $x_{i}$ and later time, $t$. Moreover, the amplitudes of the latter fluctuations are diminished relative to the amplitudes of the former fluctuations by factors $f^{3}$ and $f$. Thus, relative to a disturbance in the stationary fluid the corresponding disturbance in the expanding fluid is gradually enlarged in space, stretched out in time, and the density and velocity fluctuations are gradually diminished. As $T \rightarrow \bar{t}$ or $t \rightarrow \infty$ this relative enlargment in space and stretching out in time both approach infinity, and all density and velocity fluctuations approach zero.

The stretching out in time can be further illustrated as follows. Let $L(T)=T_{1}-T$ be a given period of the first solution, then the corresponding period of the second solution, $l(t)=t_{1}-t$, is by (4.4) and (4.5)

$$
l(t)=\frac{L(T)}{\left(1-T_{1} / \bar{t}\right)(1-T / \bar{t})} .
$$


If $L(t) \ll \bar{t}$ then

$$
l(t)=\frac{L(T)}{(1-T / \bar{t})^{2}} .
$$

Thus, all periods of the second solution at time, $t$, are stretched out relative to the corresponding periods of the first solution at time, $T$, as $T$ and $t$ increase above zero. Moreover, the relative stretching out approaches infinity as $T \rightarrow \bar{t}$ and $t \rightarrow \infty$.

The enlargement in space can be further illustrated as follows. If $Q_{i}(T)$ is the radius vector to a specific fluid element of the solution, $\widehat{\rho}, \widehat{v}_{i}$ at time $T$ and $q_{i}(t)$ is the radius vector to the corresponding fluid element of the solution, $\rho, v_{i}$ at time $t$, then (4.2) can be expressed as

or

$$
\frac{d q_{i}(t)}{d t}-\frac{q_{i}(t)}{t+\bar{t}}=\frac{1}{1+t / \bar{t}} \frac{d Q_{i}(T)}{d T} .
$$

$$
\frac{d\left[q_{i}(t) /(1+t / \bar{t})\right]}{d t}=\frac{1}{(1+t / \bar{t})^{2}} \frac{d Q_{i}(T)}{d T}
$$

or by (3.4) and (3.5)

or

$$
\frac{d\left[q_{i}(t) /(1+t / \bar{t})\right]}{d t}=\frac{d Q_{i}(T)}{d t}
$$

or by the statement following $(4.5)$

$$
q_{i}(t)=(1+t / \bar{t}) Q_{i}(T)
$$

$$
q_{i}(t)=\frac{Q_{i}(T)}{1-T / \bar{t}} .
$$

Note that $q_{i}(0)=Q_{i}(0)$. Thus, (4.12) gives the position, $q_{i}(t)$, of a fluid element of the second solution at time $t$ in terms of the position, $Q_{i}(T)$, of the fluid element of the first solution at time $T$ with which it coincides initially, at $T=t=0$. One denotes these two elements as corresponding elements. The relationship (4.12) is valid also for the vectors $D_{i}(T)$ and $d_{i}(t)$ separating any corresponding fluid element pairs, i.e.,.

$$
d_{i}(t)=\frac{D_{i}(T)}{1-T / \bar{t}}
$$

The enlargement in space is clearly illustrated by (4.12) and (4.13).

By (4.1) and (4.2) one notes that if $\hat{\rho}-\bar{\rho}, \widehat{v}_{i}$ is an isolated disturbance, i.e., $\widehat{\rho}-\bar{\rho}$ and $\widehat{v}_{i}$ vanish outside a closed region at time $T$, then the corresponding disturbance is also isolated, i.e., $\rho-\rho^{*}$ and $v_{i}-v_{i}^{*}$ vanish outside the corresponding closed region at time $t$. In Appendix A one shows that the mass, $M(T)$, associated with an isolated disturbance $\hat{\rho}-\bar{\rho}, \widehat{v}_{i}$ at time $T$ is related to the mass, $m(t)$, of the corresponding isolated disturbance $\rho-\rho^{*}, v_{i}-v_{i}^{*}$ at time $t$ as

$$
m(t)=M(T) .
$$

One also shows there that if $H_{i}(T)$ is the radius vector to the mass center of the isolated disturbance $\hat{\rho}-\bar{\rho}, \widehat{v}_{i}$ at time $T$, then the radius vector, $h_{i}(t)$, to the mass center of the corresponding isolated disturbance $\rho-\rho^{*}, v_{i}-v_{i}^{*}$ at time $t$ is given by

$$
h_{i}(t)=\frac{H_{i}(T)}{1-T / \bar{t}} .
$$


If $d\left[H_{i}(T)\right] / d T$ is a constant, $\bar{k}_{i}$, so that

$$
H_{i}(T)=\bar{k}_{i} T+H_{i}(0)
$$

where $H_{i}(0)$ is the radius vector to the mass center at $T=0$, then by (4.15)

$$
\frac{d h_{i}(t)}{d T}=\frac{\bar{k}_{i}}{1-T / \bar{t}}+\frac{\bar{k}_{i} T+H_{i}(0)}{\bar{t}(1-T / \bar{t})^{2}}
$$

or

$$
\frac{d h_{i}(t)}{d T}=\frac{\bar{k}_{i} \bar{t}+H_{i}(0)}{\bar{t}(1-T / \bar{t})^{2}}
$$

By (4.4) and (5.5)

$$
\frac{d h_{i}(t)}{d t}=\bar{k}_{i}+\frac{H_{i}(0)}{\bar{t}}
$$

Thus, if the velocity of the mass center relative to inertial space of the isolated disturbance $\widehat{\rho}-\bar{\rho}, \widehat{v}_{i}$ is constant, then the same is true for the corresponding isolated disturbance $\rho-\rho^{*}, v_{i}-v_{i}^{*}$. Moreover, the velocity $d\left[h_{i}(t)\right] / d t$ equals $\bar{k}_{i}$ plus the undisturbed expansion velocity at the radius vector $H_{i}(0)$ at the time $T=t=0$. By (4.14) and (4.19) one finds that if the momentum, $M(T) d\left[H_{i}(T)\right] / d T$, relative to inertial space of an isolated disturbance $\widehat{\rho}-\bar{\rho}, \widehat{v}_{i}$ is constant, then the same is true for the momentum, $m(t) d\left[h_{i}(t)\right] / d t$ relative to inertial space of the corresponding isolated disturbance $\rho-\rho^{*}, v_{i}-v_{i}^{*}$.

One notes that the radius vector to corresponding nodes along rays of a train of infinitesimal dilatational waves also satisfy (4.15), i.e., if $H_{i}(T)$ is the position of a given node along a ray of such waves propagating in the stationary fluid at time $T$, then $h_{i}(t)$ is the position of the corresponding node in the expanding fluid at time $t$. Since for such waves $H_{i}(T)$ satisfies (4.16), $h_{i}(t)$ satisfies (4.19). Thus, along any ray of such waves in the expanding fluid a node ahead moves with slightly greater constant velocity than the node behind, and all wave lengths expand gradually with time.

Finally, one shows in Appendix B that if $\widehat{\rho}, \widehat{v}_{i}$ is itself a homogeneous and isotropic expansion, namely, (1.4) and (1.5) with $\bar{t}$ replaced by the constant $t_{1}$, then the transformation (3.1) to (3.5) yields the same kind of expansion, namely, (1.4) and (1.5) with $\bar{t}$ replaced by the constant $\bar{t} \bar{t}_{1} /\left(\bar{t}+\bar{t}_{1}\right)$.

Appendix A. If $\hat{\rho}-\bar{\rho}, \widehat{v}_{i}$ is an isolated disturbance so that $\hat{\rho}\left(X_{1}, X_{2}, X_{3}, T\right)-\bar{\rho}$ and $\widehat{v}_{i}\left(X_{1}, X_{2}, X_{3}, T\right)$ at time $T$ vanish outside a closed region, $W(T)$, then the mass, $M(T)$, associated with the disturbance at time $T$ is given by

$$
M(T)=\iiint_{W^{\prime}(T)}\left[\widehat{\rho}\left(X_{1}, X_{2}, X_{3}, T\right)-\bar{\rho}\right] d X_{1} d X_{2} d X_{3} .
$$

By (3.1), (3.3), and (3.5) one finds

$$
M(T)=\iiint_{w^{\prime}(t)}\left[\rho\left(x_{1}, x_{2}, x_{3}, t\right)-\rho^{*}(t)\right] d x_{1} d x_{2} d x_{3}
$$

where the integration is performed at the fixed value of $t$ corresponding to the fixed 
value of $T$ in (A-1), and where $w(t)$ is the region outside of which $\rho\left(x_{1}, x_{2}, x_{3}, t\right)-$ $\rho^{*}(t)$ vanishes at time $t$. Now, the right side of (A-2) is the expression for the mass, $m(t)$, associated with the isolated disturbance $\rho-\rho^{*}, v_{i}-v_{i}^{*}$ at time $t$, i.e., one finds that

$$
m(t)=M(T)
$$

The radius vector, $H_{i}(T)$, to the mass center of the isolated disturbance $\widehat{\rho}\left(X_{1}, X_{2}, X_{3}, T\right)-\bar{\rho}, \widehat{v}_{i}\left(X_{1}, X_{2}, X_{3}, T\right)$ at any fixed time $T$ is given by

$$
H_{i}(T)=\iiint_{W(T)} X_{i}\left[\widehat{\rho}\left(X_{1}, X_{2}, X_{3}, T\right)-\bar{\rho}\right] d X_{1} d X_{2} d X_{3} .
$$

By (3.1), (3.3), and (3.5) one finds

$$
(1+t / \bar{t}) H_{i}(T)=\iiint_{u^{\prime}(t)} x_{i}\left[\rho\left(x_{1}, x_{2}, x_{3}, t\right)-\rho^{*}(t)\right] d x_{1} d x_{2} d x_{3}
$$

where the integration is performed at the fixed value of $t$ corresponding to $T$ in (A-4). Now, the right side of $(\mathrm{A}-5)$ is the radius vector, $h_{i}(t)$, to the isolated disturbance $\rho\left(x_{1}, x_{2}, x_{3}, t\right)-\rho^{*}(t), v_{i}\left(x_{1}, x_{2}, x_{3}, t\right)$ at time $t$, i.e., one finds

$$
h_{i}(t)=(1+t / \bar{t}) H_{i}(T)
$$

or by the statement following (4.5)

$$
h_{i}(t)=\frac{H_{i}(T)}{1-T / \bar{t}} .
$$

Appendix B. One assumes here that $\widehat{\rho}, \widehat{v}_{i}$ is itself a homogeneous and isotropic expansion given by

$$
\begin{gathered}
\hat{\rho}=\frac{\bar{\rho}}{\left(1+t / \bar{t}_{1}\right)^{3}} \\
\widehat{v}_{i}=\frac{x_{i}}{t+\bar{t}_{1}}
\end{gathered}
$$

which is identical to (1.4) and (1.5) except that $\bar{t}$ is replaced by the constant $\bar{t}_{1}$. The transformation (3.1) to (3.5) now yields

$$
\begin{gathered}
\rho=\frac{\bar{\rho}}{\left(1+T / \bar{t}_{1}\right)^{3}(1+t / \bar{t})^{3}} \\
v_{i}=\frac{X_{i}}{\left(T+\bar{t}_{1}\right)(1+t / \bar{t})}+\frac{x_{i}}{t+\bar{t}} .
\end{gathered}
$$

By (3.3) to (3.5) and some primitive manipulation (B-3) and (B-4) can be given the forms

$$
\begin{gathered}
\rho=\frac{\bar{\rho}}{\left[1+t /\left(\bar{t}_{1} /\left(t+t_{1}\right)\right)\right]^{3}} \\
v_{i}=\frac{x_{i}}{t+\bar{t}_{1} /\left(\bar{t}_{1}+\bar{t}_{1}\right)} .
\end{gathered}
$$


Thus, when the expansion (B-1) and (B-2) is superimposed on the expansion (1.4) and (1.5), then the result is simply an expansion of the same kind, namely, the expansion (1.4) and (1.5) with $\bar{t}$ replaced by the constant $\bar{t} \bar{t}_{1} /\left(\bar{t}+\bar{t}_{1}\right)$.

\section{REFERENCES}

[1] J. W. S. Rayleigh, The Theory of Sound, Dover Publications, N.Y., 1945

[2] M. W. Zemansky, Heat and Thermodynamics, McGraw-Hill, Inc., N.Y., 1943 